\title{
In situ photoswitching of spirorhodamine isomers in solid state
}

\author{
J. Alday, ${ }^{1}$ M. Michel Torino, ${ }^{1}$ L. Alvarez, ${ }^{2}$ M. G. Lagorio, ${ }^{1}$ C. Huck Iriart, ${ }^{3}$ S. Suarez ${ }^{1}$ \\ ${ }^{1}$ INQUIMAE, CONICET and Departamento de Química Inorgánica, Analítica y Química Física, Facultad de Ciencias Exactas y \\ Naturales, Universidad de Buenos Aires, CABA, Argentina, \\ ${ }^{2}$ IIB, UNSAM, 25 de mayo y Francia, San Martín Buenos Aires, Argentina, \\ ${ }^{3}$ ECyT, UNSAM, 25 de mayo 1169, San Martín, Buenos Aires, Argentina. \\ seba@qi.fcen.uba.ar
}

At present, the functional materials structurally switchable by stimuli such as heat, the addition of cations, changes of $\mathrm{pH}$, pressure, or light are the motive of innumerable studies to be ideal models to investigate the relation structure-function and new properties derived from that change. In this work, we studied a family of spirorhodamines (SRAs) in solid-state photochemical reactions. These are photochromic molecules with a switching mechanism based on the differences in the fundamental electronic state between isomers.[1] It involves changes in the molecule structure and is thermally reversible.[2,3] In this work, assuming as the hypothesis that in solidphase the permanence time in the optically active isomer is associated with its structural characteristics, a family of compounds modifying the substituent was synthesized.

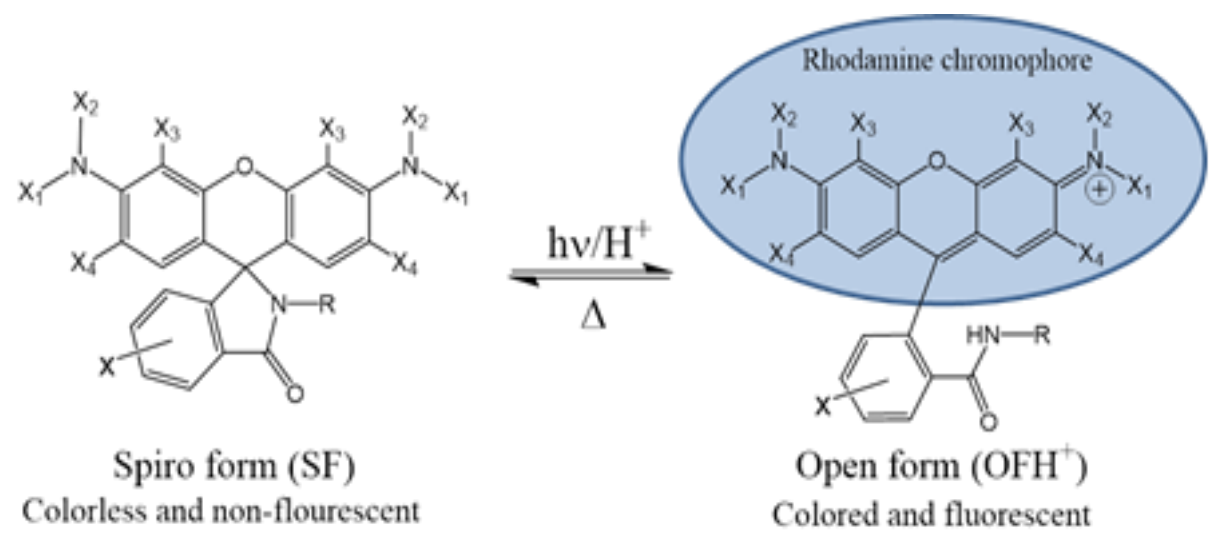

Figure 1. General structure of spirorhodamines. The equilibrium responsible for the appearance of color is also shown. The forward reaction can be photo-induced with UV light, or by proton addition.

These equilibria were characterized in solid-state by reflection, absorption and emission fluorescence spectroscopy, single-crystal Xray diffraction,[4] atomic force microscopy coupled to infrared spectroscopy [4] and computational calculations, evaluating the changes produced after irradiating the corresponding close isomer with ultraviolet light for each compound.

[1] Dürr, H., Bouas-Laurent, H. (2003) Photochromism: Molecules and Systems, Eds.

[2] Di Paolo, M., Bossi, M. L., Baggio, R. and Suarez, S. A. (2016) Acta Crystallogr. Sect. B Struct. Sci. Cryst. Eng. Mater., 72 , 684.

[3] Di Paolo, M., Boubeta, F., Alday, J., Michel Torino, M., Aramendía, P., Suarez, S.* and Bossi, M.* (2019) J. Photochem. Photobiol. A,. 384, 112011.

[4] Brazilian Synchrotron Light Laboratory (LNLS) beamlines MX2 and IR1.

Keywords: Photoswitching, spirorhodamines, solid-state, synchrotron, single-crystal X-ray diffraction.

This work was supported by Universidad de Buenos Aires, Argentina. The authors would like to thank the economic support of the LNLS. 